\title{
Formación en Comunicación e Internacionalización
}

\section{Communication and Internationalization Curriculum}

Genny Elizabeth Góngora Cuevas*

Universidad Anáhuac México genny.gongora@anahuac.mx

http://orcid.org/0000-0003-3059-4818

Av. Universidad Anáhuac núm. 46, Col. Lomas Anáhuac, Huixquilucan, Estado de México, C.P. 052786, México

Editor: Rogelio del Prado Flores

Fecha de recepción: 24 de junio de 2021

https://doi.org/10.36105/stx.2021edesp50aniv.08

\section{Resumen}

A partir de su creación en I964, la Universidad Anáhuac México ha tenido la vocación de formar líderes que influyan en la sociedad a través del ejercicio de su profesión, es por eso que en las aulas de la Facultad de Comunicación han pasado grandes figuras de los medios de comunicación nacional. Con los movimientos de la población buscando nuevas y mejores oportunidades de trabajo se agudiza la necesidad de mantener una oferta educativa vanguardista que permita la rápida incorporación al mundo laboral y al mismo tiempo responda a los requerimientos futuros de los empleadores.

La Universidad en busca de la excelencia, ha optado por acreditaciones de los programas de estudio con acreditadoras nacionales e internacionales y está presente en los rankings internacionales que miden la calidad de los programas educativos. Estas acreditaciones le permiten a la Facultad competir en la arena con los mejores programas de comunicación en el mundo.

Palabras clave: currícula, internacionalización, comunicación, educación superior, acreditación de programas educativos.

\footnotetext{
* Doctora en Gestión Estratégica y Políticas de desarrollo, maestra en Enseñanza Efectiva. Fue directora general adjunta de la Dirección de Centros de Formación para el Trabajo de la Subsecretaría de Educación Media superior de la Secretaría de Educación Pública. Participó en mesas de trabajo ante organismo como la OIT, BID, OCED, OEA. Actualmente es coordinadora de acreditaciones y vinculación internacional para la Facultad de Comunicación de la Universidad Anáhuac México.
} 
ABSTRACT

Since its creation in 1964, the Universidad Anáhuac Mexico has had the vocation of training leaders who influence society through the exercise of their profession that is why great figures from the national media have passed in the classrooms of the Faculty of Communication. With the movements of the population seeking new and better job opportunities, the need to maintain an avant-garde educational offer that allows rapid incorporation into the world of work and at the same time responds to the future requirements of employers is becoming more acute.

The University in search of excellence has opted for accreditation of study programs national and international, the Universidad Anáhuac México is present in international rankings that measure the quality of educational programs. These accreditations allow the Faculty to compete in the arena with the best communication programs in the world.

Keywords: curriculum, internationalization, communication, higher education, accreditation of education programs.

\section{INTRODUCCIÓN}

En el mundo laboral actual existe una competencia cada vez mayor por los puestos de trabajo donde las personas con más cualificaciones son las que se colocan de manera exitosa en el trabajo, en ese sentido la Universidad Anáhuac México está entre las tres primeras universidades en empleabilidad de sus egresados de acuerdo al ranking de empleabilidad QS 2020.

La Universidad Anáhuac México mantiene en constante renovación su currícula para formar de manera integral a los alumnos en las necesidades futuras que el campo laboral les demandará en su momento de egreso.

Este documento es una revisión documental de los rankings internacionales, criterios de acreditación de diferentes organismos, así como las acciones de la Universidad Anáhuac México para estar dentro de las mejores universidades de México.

\section{CONCEPTOS DE INTERNACIONALIZACIÓN EN EL MUNDO}

La Asociación Internacional de Universidades (IAU) de la Unesco considera la internacionalización como proceso intencional, un componente de la solidaridad internacionaly 
un instrumento de primer orden para incrementar la calidad la educación e investigación para los alumnos y los docentes y lograr una contribución significativa para la sociedad (IAU, s.f.).

En palabras de Tünnermann (20I8):

No cabe confundir globalización con internacionalización. La globalización es el flujo de tecnologías, de economía, conocimiento, etc., a través de las fronteras. Cada país se inserta en ella según su nivel de desarrollo, competitividad y adelanto de sus sistemas de educación, científico-tecnológicos y de innovación. La internacionalización de la educación superior es una de las maneras de responder a los impactos de la globalización, con pleno respeto a la identidad nacional. (p. 20)

Por tanto, la internacionalización es parte del proceso de globalización que permite a los estudiantes obtener las competencias para incorporarse a un mercado de trabajo dentro y fuera de su propio país.

La OMC (2000) ha añadido a la lista los servicios educativos, llamándolos mercado de la educación y dividiéndolos en cinco grandes categorías: educación primaria, educación secundaria, superior, para adultos y otro tipo de educación; lo que conlleva a generar nuevas regulaciones para este sector ya que es importante ver a la educación como un mercado en el cual hay que satisfacer al cliente (estudiante) en términos de su mejor estancia dentro del campus o en una plataforma en línea, a distancia o semipresencial. La educación en línea ha favorecido también la internacionalización del currículum y el incremento de estudiantes extranjeros matriculados en las universidades.

De acuerdo con Mazzarol y Soutar (1999), el mercado mundial de la educación internacional se mide en el número de estudiantes que se matriculan en una universidad fuera de su país. El documento de Política para el cambio y el desarrollo en la educación superior, que la Unesco elaboró para la Conferencia Mundial sobre la Educación Superior en París, deja una idea clara del concepto de internacionalización:

La internacionalización cada vez mayor de la educación superior es en primer lugar, y ante todo, el reflejo del carácter mundial del aprendizaje y la investigación. Ese carácter mundial se va fortaleciendo gracias a los procesos actuales de integración económica y política, por la necesidad cada vez mayor de comprensión intercultural y por la naturaleza mundial de las comunicaciones modernas, los mercados de consumidores actuales, etc. El incremento permanente del número de estudiantes, profesores e investigadores que estudian, dan cursos, investigan, viven y comunican en un marco internacional es buena muestra de esta nueva situación general, a todas luces benéfica. (Unesco, 1995, p. I) 
La Declaración Mundial sobre la Educación Superior en el siglo XXI: Visión y Acción (Unesco, 1998) menciona una serie de acciones que deberán emprenderse en el plano de lo internacional, a continuación se enuncian textualmente:

- Deberá concebirse la cooperación como parte integrante de las misiones institucionales de los establecimientos y sistemas de educación superior. Las organizaciones intergubernamentales, los organismos donantes y las organizaciones no gubernamentales deberán ampliar su acción para desarrollar proyectos de cooperación interuniversitaria en particular mediante el hermanamiento de instituciones basados en la solidaridad y la asociación, con objeto de acortar la distancia que separa a países ricos y países menos adelantados en los ámbitos cruciales de la producción y aplicación de los conocimientos. Cada establecimiento de enseñanza superior deberá prever la creación de una estructura o un mecanismo apropiados para promover y organizar la cooperación internacional.

- La Unesco y otras organizaciones intergubernamentales y no gubernamentales que actúan en el campo de la educación superior, los Estados mediante sus programas de cooperación bilateral y multilateral, la comunidad universitaria y todos los interlocutores interesados de la sociedad deberán promover la movilidad universitaria internacional como medio de hacer progresar el saber y de compartirlo, a fin de instaurar y fomentar la solidaridad, principal elemento de la futura sociedad mundial del saber, inclusive mediante un fuerte apoyo al plan de trabajo conjunto (1999-2005) de los seis comités intergubernamentales encargados de la aplicación de los convenios regionales sobre la convalidación de estudios, diplomas y títulos de enseñanza superior, y por medio de una acción cooperativa en gran escala, con particular acento en la cooperación Sur-Sur, las necesidades de los países menos adelantados y de los pequeños Estados, que o carecen de establecimientos de educación superior o tienen muy pocos.

- Los establecimientos de educación superior de los países industrializados deberán esforzarse por concertar acuerdos de cooperación internacional con establecimientos homólogos de países en desarrollo, en particular con los de los países menos adelantados. En el marco de esa cooperación los establecimientos deberán esforzarse por garantizar un reconocimiento justo y razonable de los estudios cursados en el extranjero. La Unesco deberá adoptar iniciativas para promover el desarrollo de la educación superior en todo el mundo, fijando objetivos concretos que puedan plasmarse en resultados tangibles. Una de las modalidades consistiría en ejecutar proyectos en las diferentes regiones, redoblando esfuerzos por 
crear o consolidar centros de excelencia en los países en desar rollo, especialmente mediante el Programa UNITWIN y de Cátedras Unesco, fundándose en las redes de instituciones de enseñanza superior nacionales, regionales de internacionales.

- La Unesco, junto con todos los interlocutores interesados de la sociedad, también deberá tomar medidas para paliar los efectos negativos de la fuga de cerebros y sustituirla por un proceso dinámico de recuperación de los mismos. En todas las regiones del mundo se necesita un análisis global de las causas y consecuencias de la fuga de cerebros. Deberá ponerse en marcha una vigorosa campaña, mediante el esfuerzo concertado de la comunidad internacional y basada en la solidaridad universitaria, que debería centrarse en el regreso al país de origen de universitarios expatriados y en la participación de voluntarios universitarios (profesores recién jubilados o jóvenes universitarios en principio de carrera) que deseen enseñar e investigar en establecimientos de educación superior de países en desarrollo. Al mismo tiempo, es fundamental apoyar a los países en desarrollo en sus esfuerzos para construir y consolidar sus propias capacidades educativas. (Unesco, I998, pp. I2I-I22)

En la Conferencia Mundial sobre la Educación Superior-20og: La nueva dinámica de la educación superior y la investigación para el cambio social y el desarrollo realizada en París en julio de 2009, queda de manifiesto nuevamente la importancia de la internacionalización de docentes y alumnos y la protección de la fuga de cerebros o competencias (Unesco, 2009).

La internacionalización se puede concebir en tres vertientes:

- Desde el punto de vista financiero, el captar más estudiantes que paguen por la matrícula completa (Mcnamara y Harris, 1997). Para lo cual Fallon y Brown (1999) destacan el carácter comercial de esta vertiente considerando que las universidades promoverán sus bondades hacia el exterior dando el plus del ambiente multicultural, adaptando sus programas para la complacencia o satisfacción del cliente (Ackers, 1997).

- Enfoque de "infusión" el cual se caracteriza por incluir en el currículo contenidos que reflejen la realidad profesional en diferentes culturas con el objetivo de desarrollarse social y profesionalmente en un contexto internacional y multicultural (Van Der Wende, 2000).

- Pedagogía culturalmente inclusiva en el centro del currículum, en esta vertiente la importancia central está en el desarrollo de contenidos que permitan al alumno te- 
ner un conjunto de datos referentes a la globalización (Rizvi, 2007), sin embargo, existen autores que consideran la capacitad analítica, de cuestionarse, identificar fuentes de conocimiento, evaluar y justificar las mismas como competencias esenciales a desarrollar en el alumno (Ziguras y Rizvi, 200I).

Entrar a un ambiente de internacionalización supone un reto mayor para los profesores que deberán entender los diferentes contextos de los que provienen sus estudiantes, así como aplicar estrategias docentes para lograr pensamientos de orden superior. De acuerdo con De Vita y Case (2003) y Crisan-Mitra y Borza (2015) al interactuar un alumno con contenidos multiculturales y al estar en contacto directo con estudiantes de diferentes partes del mundo puede ayudar a crear ambientes de aprendizaje en los cuales los estudiantes estén realmente expuestos a un perspectiva multicultural.

De acuerdo con el Instituto de Educación Internacional (202I), en el reporte de $2017 \mathrm{del}$ Proyecto Atlas, la distribución de estudiantes que están cursando algún tipo de programa universitario en los 24 países adscritos se muestra en el Cuadro I.

CUADRO 1. PAÍSES QUE RECIBEN ALUMNOS EXTRANJEROS (2017)

\begin{tabular}{|c|c|c|c|c|c|}
\hline \multicolumn{6}{|c|}{ AMÉRICA DEL NORTE } \\
\hline \multicolumn{2}{|c|}{ CANADÁ } & \multicolumn{2}{|c|}{ ESTADOS UNIDOS } & \multicolumn{2}{|c|}{ MÉXICO } \\
\hline China & $132,345.00$ & China & $350,734.00$ & $\begin{array}{l}\text { Estados } \\
\text { Unidos }\end{array}$ & $4,213.00$ \\
\hline India & $76,530.00$ & India & $186,264.00$ & Colombia & $2,805.00$ \\
\hline Francia & $20,790.00$ & Corea del Sur & $56,660.00$ & Francia & $1,884.00$ \\
\hline \multirow{2}{*}{ Estados Unidos } & \multirow{2}{*}{$12,915.00$} & Arabia Saudita & $61,287.00$ & Alemania & $1,282.00$ \\
\hline & & Canadá & $26,973.00$ & España & $1,231.00$ \\
\hline \multicolumn{6}{|c|}{ EUROPA } \\
\hline \multicolumn{2}{|c|}{ FINLANDIA } & \multicolumn{2}{|c|}{ PAÍSES BAJOS } & \multicolumn{2}{|c|}{ FRANCIA } \\
\hline Rusia & $3,243.00$ & Alemania & $22,189.00$ & China & $25,388.00$ \\
\hline Vietnam & $2,529.00$ & China & $4,347.00$ & Marruecos & $25,223.00$ \\
\hline
\end{tabular}


FORMACIÓN EN COMUNICACIÓN E INTERNACIONALIZACIÓN - GENNY GÓNGORA

\begin{tabular}{|c|c|c|c|c|c|}
\hline Francia & $2,405.00$ & Italia & $3,347.00$ & Algeria & $16,558.00$ \\
\hline & & Bélgica & $2,976.00$ & Túnez & $8,955.00$ \\
\hline Estados Unidos & $1,657.00$ & Reino Unido & $2,778.00$ & Senegal & $7,439.00$ \\
\hline \multicolumn{2}{|c|}{ ALEMANIA } & \multicolumn{2}{|c|}{ RUSIA } & \multicolumn{2}{|c|}{ ESPAÑA } \\
\hline China & $25,388.00$ & Kazajistán & $73,445.00$ & Italia & $13,157.00$ \\
\hline India & $13,537.00$ & Ucrania & $23,217.00$ & Francia & $10,065.00$ \\
\hline Rusia & $11,413.00$ & China & $22,529.00$ & $\begin{array}{l}\text { Estados } \\
\text { Unidos }\end{array}$ & $7,047.00$ \\
\hline Francia & $10,129.00$ & Uzbekistán & $21,577.00$ & México & $6,919.00$ \\
\hline Austria & $7,330.00$ & Turkmenistán & $18,934.00$ & Alemania & $6,699.00$ \\
\hline \multicolumn{3}{|c|}{ SUECIA } & \multicolumn{3}{|c|}{ REINO UNIDO } \\
\hline \multicolumn{2}{|l|}{ Francia } & $11,771.00$ & \multicolumn{2}{|l|}{ China } & $97,850.00$ \\
\hline \multicolumn{2}{|l|}{ Alemania } & $3,625.00$ & \multicolumn{2}{|l|}{ Estados Unidos } & $28,125.00$ \\
\hline \multicolumn{2}{|l|}{ Finlandia } & $2,495.00$ & \multicolumn{2}{|l|}{ Malasia } & $18,400.00$ \\
\hline \multicolumn{2}{|l|}{ China } & $2,374.00$ & \multicolumn{2}{|l|}{ Alemania } & $18,205.00$ \\
\hline \multicolumn{2}{|l|}{ India } & $1,316.00$ & \multicolumn{2}{|l|}{ India } & $18,015.00$ \\
\hline \multicolumn{6}{|c|}{ ASIA } \\
\hline \multicolumn{2}{|c|}{ CHINA } & & & & \\
\hline Corea del Sur & $70,540.00$ & China & $75,262.00$ & Nepal & $9,574.00$ \\
\hline Estados Unidos & $23,838.00$ & Vietnam & $28,571.00$ & Afganistán & $4,404.00$ \\
\hline Tailandia & $23,838.00$ & Corea del sur & $13,571.00$ & Bután & $2,925.00$ \\
\hline Pakistán & $18,626.00$ & Nepal & $13,458.00$ & Nigeria & $2,090.00$ \\
\hline India & $18,626.00$ & Taiwán & $6,401.00$ & Sudán & $2,059.00$ \\
\hline
\end{tabular}




\begin{tabular}{|c|c|c|c|}
\hline \multicolumn{4}{|c|}{ OCEANÍA } \\
\hline \multicolumn{2}{|c|}{ AUSTRALIA } & \multicolumn{2}{|c|}{ NUEVA ZELLANDA } \\
\hline China & $114,006.00$ & China & $31,075.00$ \\
\hline India & $44,775.00$ & Vietnam & $19,585.00$ \\
\hline Nepal & $15,211.00$ & Estados Unidos & $4,445.00$ \\
\hline Malasia & $14,721.00$ & Malasia & $2,725.00$ \\
\hline Vietnam & $13,949.00$ & Filipinas & $2,160.00$ \\
\hline
\end{tabular}

FUENTE: ELABORACIÓN PROPIA CON DATOS DEL INSTITUTO dE EdUCACIÓN INTERNACIONAL, 2021.

\section{internacionalización en América Latina}

En América Latina, según Didou (2005), los procesos de integración se plantearon hacia la primera mitad del siglo XX, acompañados también de algunos desarrollos universitarios con algunas particularidades, las redes regionales de instituciones respondían a una serie de motivaciones con sentido regional, subregional o nacional: el mejoramiento de la calidad institucional, la promoción de la movilidad de estudiantes y profesores, la socialización de la investigación y la formación continua, la internacionalización de los currículos debido a la aceptación que los estudiantes ya no se preparaban solo para los mercados nacionales, sino para los mercados regionales y globales. A partir del Informe Bricall (2000), se reconoce que la cooperación entre las universidades favorecía la renovación de la formación adquirida y posibilidad del reconocimiento de los títulos profesionales en espacios geográficos más amplios.

Las universidades latinoamericanas no están exentas de las presiones generadas por los diferentes acuerdos de comercio para liberar los servicios de la educación; las universidades más grandes en cada uno de los países latinos son públicas, lo que implica que el financiamiento viene de una cuenta pública; se ha estado tratando de cambiar el paradigma al ofrecer la educación a través de las Tecnologías de la Información y Comunicación para abrir el abanico de opciones y de estudiantes que se matriculan en la universidad, representando los alumno extranjeros una fuente adicional de ingresos.

De acuerdo con lo que se menciona en Bricall (2000), las universidades pueden ver en la educación a distancia una forma de generar economías de escala. 
Cada vez es más frecuente que las universidades de Latinoamérica se preocupen por la calidad como una forma de afianzar como un jugador más a sus programas de estudio en el mercado internacional, y esto se puede comprobar fácilmente al ver la lista de programas acreditados en organismo como: ABET, ACEJMC, AACSB, AMBA, RIBA, entre otras. Sin embargo, las estadísticas de los alumnos de América Latina que prefieren estudiar en Estados Unidos o en Europa va en aumento.

En México, las estadísticas registradas en 2017 por la Dirección General de Educación Superior perteneciente a la Secretaría de Educación Pública (SEP, 20I7) indican que un total de 5 millones de mexicanos cursan sus estudios en el extranjero, de los cuales $17 \%$ lo hace en Estados Unidos.

De los países de America Latina con estudiantes en Estados Unidos, durante 2018 se puede observar un crecimiento en los estudiantes de Brasil en un I3.I\%, Chile en 6.2\% y Argentina en $6.7 \%$, así como una caída de los estudiantes Venezolanos en $-8.7 \%$. Cabe señalar que en cuanto a los estudiantes de México en Estados Unidos hubo una baja del 6\% con relación al año 2017 (SEVIS, 20I8). Como estrategia para lograr la internacionalización de currículum y captar estudiantes para sus universidades algunos países de América Latina han dado pasos firmes, de tal manera que por ejemplo en el año 2005 varios países ya eran miembros del International Network for Quality Assurance Agencies in Higher Education (INQAAHE) (Cuadro 2).

CUADRO 2. MIEMBROS DE AMÉRICA LATINA EN LA INTERNATIONAL NETWORK FOR QUALITY ASSURANCE AGENCIES IN HIGHER EDUCATION (INQAAHE)

\begin{tabular}{|l|c|}
\hline \multicolumn{1}{|c|}{ MIEMBRO } & PAís \\
\hline Comisión Nacional de Evaluación y Acreditación Universitaria (CONEAU) & Argentina \\
\hline Comisión Nacional de Acreditación de Pregrado (CNAP) & \\
\hline Consejo Superior de Educación (CSE) & Chile \\
\hline Consejo Nacional de Acreditación & Colombia \\
\hline
\end{tabular}




\begin{tabular}{|c|c|}
\hline \multicolumn{1}{|c|}{ MIEMBro } & PAís \\
\hline Comisión para la Acreditación de la Educación Superior (COPAES) & México \\
\hline Comisión Nacional de Evaluación (CONAEVA) & \\
Consorcio de Universidades compuesto por: & Perú \\
- Univificia Universidad Católica del Perú & \\
- Universidad del Pacífico & \\
\hline
\end{tabular}

FUENTE: ELABORACIÓN PROPIA CON BASE EN IN QAAHE, 2005.

\section{INTERNACIONALIZACIÓN EN MÉXICO}

México tiene un gran reto como país proveedor de servicios educativos, la cada vez más restrictiva política migratoria y de visado de los Estados Unidos aunado a la economía mundial hace que algunos países volteen sus ojos a ofertas más asequibles tomando en consideración que un semestre de estudios en una universidad pública en Estados Unidos tiene un costo mínimo de 20000.00 dólares para los estudiantes extranjeros en universidades públicas o más de 70 o००.०० dólares en universidades de prestigio (Collegue Board, 2019).

El reto de la internacionalización no es un paradigma nuevo en nuestro país, desde la década de los años noventa se empezó a mirar hacia el extranjero como una nueva forma de crear capital humano competente y poder sacar adelante al país, esto se ve palpable al analizar el Plan Nacional de Desarrollo (PND) de los sexenios pasados. Al revisar el PND de 20I3-20I8 se puede observar que se expresa como objetivo claro el tener una educación de calidad y un México con responsabilidad social (Gobierno de la República, 2013). Lo anterior se tradujo claramente en el Plan sectorial de educación 20I3-20I8 en dos estrategias concretas del objetivo 2: "Fortalecer la calidad y pertinencia de la educación media superior, 
superior y formación para el trabajo, a fin de que contribuyan al desarrollo de México" bajo los numerales:

- 2.3.8 Apoyar nuevos modelos de cooperación académica para la internacionalización de la educación superior.

- 2.3.9 Promover que más egresados cuenten con capacidades suficientes para ser admitidos en los mejores programas de posgrado de México y el mundo.

El Plan Nacional de Desarrollo 20 I8-2024, presentado el 30 de abril de 2019 ante la Cámara de Diputados, tiene tres ejes generales y tres ejes transversales. En el eje "Bienestar" se encuentra un objetivo y un indicador claramente identificados con la calidad de la educación Secretaría de Gobernación (2019):

- Objetivo: 2.2. Garantizar el derecho a la educación laica, gratuita, incluyente, pertinente y de calidad en todos los tipos, niveles y modalidades del Sistema Educativo Nacional y para todas las personas.

- Indicador: 2.2.I Eficiencia del Sistema Educativo, por nivel y tipo (PSE 20I32018).

Al tener una cultura de la calidad en la educación que esté reconocida por las agencias que avalan la calidad educativa en Estados Unidos se atraen más estudiantes de todas partes de América Latina y el mundo, que ven a México como una alternativa de obtener la misma calidad de educación a precios mucho más accesibles que en países europeos o en Estados Unidos y Canadá. Es por eso que la internacionalización en México se ve como una nueva forma de ser competitivos, de participar en la sociedad del conocimiento, innovar y crear tecnología.

Se puede identificar que los actores en la internacionalización en México son:

I. El gobierno mexicano:

Secretaría de Educación Pública a través de:

a) Dirección General de Educación Superior Universitaria (DGESU), depende de la Subsecretaría de Educación Superior.

b) Dirección General de Relaciones Internacionales (DGRI), que depende directamente de la oficina del Secretario de Educación. 
2. Las Instituciones de Educación Superior que se dividen en instituciones públicas y privada, según el origen de los recursos que reciben.

3. Asociaciones nacionales:

a) Asociación Nacional de Universidades e Instituciones de Educación Superior (ANUIES).

b) Federación de Instituciones Mexicanas Particulares de Educación Superior (FIMPES).

c) Asociación Mexicana para la Educación Internacional (AMPEI).

4. Organismos internacionales.

a) Banco Mundial (BM).

b) Organización para la Cooperación y el Desarrollo Económico (OCDE).

c) La Organización de las Naciones Unidas para la Educación, la Ciencia y la Cultura (Unesco).

d) Banco Interamericano de Desarrollo (BID).

De acuerdo con la publicación Education at a Glance: OECD indicators 20r6, la cual muestra estadísticas de temas de educación para sus países miembros, el promedio de estudiantes nacionales de educación superior estudiando en el extranjero es de $5.7 \%$, en este sentido destaca en primer lugar Luxemburgo con $68.4 \%$ seguido por República Eslovaca con I4.2\%, en la última posición está Estados Unidos con $0.3 \%$, la estadística para México es de $0.8 \%$. En cuanto al número de extranjeros por estudiante nacional estudiando en el extranjero destaca Australia con 20.7\%, seguido por Reino Unido, en tercer lugar está Estados Unidos y México ocupa la última posición con $0.3 \%$, el promedio para los países miembros de la OECD es de $3.2 \%$ (OECD, 2OI6).

Lo anterior significa que en México hay más estudiantes en el extranjero de los que se están atrayendo para estudiar en las universidades privadas o públicas, siendo un reto captar más alumnos que asistan a las aulas de las universidades mexicanas.

\section{LA FACULTAD DE COMUNICACIÓN, HACIA}

\section{LA INTERNACIONALIZACIÓN DEL CURRÍCULUM}

Desde su creación en 1970, la entonces Escuela de Comunicación ha tenido sus objetivos bien definidos en cuanto a la pertinencia de los planes de estudio, el perfil de egreso de 
sus alumnos y la educación basada en valores para formar líderes de acción positiva en la sociedad.

Cabe señalar que la Universidad Anáhuac México fue de las primeras universidades en ofrecer la Licenciatura en Comunicación, posicionándose en los primeros lugares por su calidad académica y pertinencia y es por eso que en el año 2007 el programa académico se sometió a escrutinio a través del proceso de acreditación del Consejo de Acreditación de la Comunicación (CONAC), logrando su primera acreditación y reacreditándose en 2012 y 20I8. Siendo ya la Facultad de Comunicación y con el ánimo de seguir con la movilidad e internacionalización de profesores y alumnos, se empezó el proceso de acreditación del programa académico con el Consejo Latinoamericano de Acreditación de la Educación en Periodismo (CLAEP), el cual es parte de la Sociedad Interamericana de Prensa (SIIP), lo que sin duda representó todo un gran reto y fue una prueba más para la calidad y pertinencia del programa académico logrando la acreditación en 2009 y la reacreditación en 2015.

Los esfuerzos de la Facultad de Comunicación en conformar un Consejo de Egresados que orientara las acciones, el apoyo de los empleadores para tener un perfil de egreso de acuerdo con los retos de la comunicación, así como el equipamiento de laboratorios, calidad de los docentes, vida universitaria, servicios a la comunidad y la incesante labor del área de investigación por generar productos con un impacto social a través del Centro de Investigación para la Comunicación Aplicada (CICA), dio como resultado que en el año de 2014 se lograra la acreditación internacional de Accrediting Council for Education in Journalism and Mass Communications (ACEJMC), siendo hasta el momento una de las dos facultades en México que cuentan con esta acreditación (la otra es el Instituto Tecnológico de Estudios Superiores de Monterrey, campus Monterrey), y junto con la Pontificia Universidad Católica de Chile son los únicos programas acreditados a nivel América Latina.

Cabe señalar que el ACEJMC tiene prestigiadas universidades con programas de comunicación reconocidos en los primeros 20 lugares a nivel mundial de acuerdo con el ranking QS Communication \& Media 2019 como son: University of Southern California (QS2), University of Texas at Austin (QS4) y Michigan State University (QSı6). La acreditación del ACEJMC permite a la Facultad de Comunicación establecer acciones en conjunto con las universidades que cuentan con la misma documentación, con lo cual estudiantes y profesores pueden participar en temas de movilidad, intercambios internacionales, investigación, entre otras acciones conjuntas, logrando de esta forma sinergias importantes para el crecimiento y posicionamiento de la Facultad de cara al futuro y los nuevos retos para la profesión.

Los planes de estudio de la Licenciatura en Comunicación han pasado de un modelo inflexible donde el estudiante no podía seleccionar sus materias y no existían salidas late- 
rales de profesionalización, hasta un currículum flexible donde el estudiante puede tomar clases en diferentes facultades dentro del campus, dentro de la Red de Universidades Anáhuac o en las universidades con las que se tenga un convenio de internacionalización. El programa académico actual permite salidas laterales dando la oportunidad a que el alumno se especialice en cualquiera de las siguientes opciones: Dirección en publicidad y comunicación empresarial, Dirección y producción cinematográfica y audiovisual, y Dirección de empresas mediáticas.

Los mejores programas académicos de comunicación aparecen en el ranking QS, por lo que de acuerdo con el análisis realizado por Tejedor y Cervi (2017) con base en el QS Communication \& Media 20I7, ubicaron los Io mejores programas de comunicación en el mundo (Cuadro 3 ) en ese momento y se determinaron las competencias del perfil de egreso que eran comunes en esas universidades (Cuadro 4).

CUADRO 3. UNIVERSIDADES Y GRADOS ANALIZADOS

\begin{tabular}{|l|l|l|}
\hline \multicolumn{1}{|c|}{ NOMBRE DE LOS ESTUDIOS } & \multicolumn{1}{c|}{ UNIVERSIDAD } & \multicolumn{1}{c|}{ PAÍs } \\
\hline Comunicación & Stanford University & EE.UU. \\
\hline Comunicación & University of California-Berkeley & EE.UU. \\
\hline Comunicación & University of Wisconsin-Madison & EE.UU. \\
\hline Periodismo & University of Wisconsin-Madison & EE.UU. \\
\hline Comunicación & University of California-Los Ángeles & EE.UU. \\
\hline Comunicación & University of Pennsylvania & EE.UU. \\
\hline Periodismo & Universidad of Southern California & EE.UU. \\
\hline Ciencias de la Comunicación & University of Amsterdam & Países Bajos \\
\hline Información y Medios & University of Amsterdam & Países Bajos \\
\hline Comunicación y Medios & Goldsmiths-University of London & Reino Unido \\
\hline Periodismo & Goldsmiths-University of London & Reino Unido \\
\hline
\end{tabular}

FUENTE: TEJEDOR Y CERVI, 2017. 
CUADRO 4. PRINCIPALES COMPETENCIAS EN LOS PLANES DE ESTUDIO EN LAS MEJORES UNIVERSIDADES DEL MUNDO

\begin{tabular}{|l|l|}
\hline \multicolumn{1}{|c|}{ COMPETENCIA } & PUNTAJE \\
\hline Conocer los fundamentos de la comunicación & 50 \\
\hline Poseer habilidades de investigación & 30 \\
\hline Desarrollar capacidades de autoreflexión & 57.1 \\
\hline Desarrollar capacidades para solucionar problemas & 21.42 \\
\hline Desarrollar capacidades para la evaluación & 30 \\
\hline Tener una capacidad crítica, analítica y ética & 100 \\
\hline Poseer habilidades comunicativas & 85.7 \\
\hline Conocer la estructura mediática & 21.42 \\
\hline Capacidad para la dirección y gestión de proyectos & 14.28 \\
\hline Poseer una actitud profesional & 30 \\
\hline Poseer capacidad de trabajo en equipo & 7.14 \\
\hline
\end{tabular}

FUENTE: TEJEDOR Y CERVI, 2017.

Es posible hacer una comparación de las competencias coincidentes en los Io mejores programas de comunicación en el mundo en 2017 y las competencias con las que se forman los alumnos de la Facultad de Comunicación, en ese sentido podemos observar que el conjunto de competencias definidas en el plan 2016 (Universidad Anáhuac México, 20I6) coinciden con las competencias de los io mejores programas de comunicación en el mundo (Cuadro 5).

\section{CUADRO 5. COMPETENCIAS DE LOS EGRESADOS ANÁHUAC DE COMUNICACIÓN}

\begin{tabular}{|l|l|}
\hline \multicolumn{1}{|c|}{ COMPETENCIAS PROFESIONALES } & COMPETENCIAS GENÉRICAS \\
\hline 1. Reconoce la importancia de la búsqueda de la verdad y del \\
pensamiento crítico, creativo e independiente en la difusión del \\
$\begin{array}{l}\text { conocimiento como elemento que guíe a las audiencias a la toma } \\
\text { de decisiones informadas y sopesadas, con el fin de construir } \\
\text { sociedades más demócratas, justas y solidarias. }\end{array}$ & 1. Capacidad crítica y autocrítica \\
\hline
\end{tabular}


2. Aplica los principios éticos profesionales, asumiéndose como un agente de transformación en una sociedad global donde se respete la diversidad e inclusión de pueblos y culturas en todas

2. Inteligencia social y emocional sus expresiones y en relación a los medios de comunicación.

3. Busca el sentido trascendente en los mensajes o productos mediáticos, publicitarios o mercadológicos que desarrolla, de tal forma que la influencia de ellos en la sociedad y la cultura sirvan a

3. Razonamiento matemático los demás para mejorar sus condiciones de vida.

4. Se comunica de manera correcta, clara, libre y responsable en cualquier forma y estilo, en lengua materna y extranjera, empleando conceptos y teorías de la comunicación apropiados para emplear los diferentes equipos, técnicas, estadísticas y

4. Cultura general mercadológicas, tecnológicas y recursos informáticos de la comunicación, a fin de apoyar al quehacer profesional.

5. Integra los conocimientos a propiados para emplear los diferentes equipos, técnicas estadísticas y mercadológicas, tecnologías y recursos informáticos de la comunicación, a fin de apoyar al

5. Compromiso con la calidad quehacer profesional.

6. Analiza y evalúa la historia y el papel de los medios a través de los distintos métodos de investigación, para el conocimiento profundo de las necesidades de las diferentes audiencias y enfatiza en el impacto de los diversos contenidos y productos mediáticos.

7. Brinda consultoría y diseña estrategias integrales de comunicación para la prevención y solución de problemas existentes en los diferentes niveles de las organizaciones, nacionales e internacionales.

8. Participa y lidera equipos de trabajo multidisciplinarios en proyectos médicos, mercadológicos, publicitarios y organizacionales a fin de provocar cambios en las audiencias y transformar positivamente la cultura y la sociedad.

9. Crea e implementa contenidos, programas, proyectos, productos mediáticos, estrategias mercadológicas y corporativas, creativas e innovadoras, que conllevan al bienestar de las audiencias y públicos a los que impactan.

COMPETENCIAS TRANSVERSALES

1. Internacionalización

10. Emprende socialmente proyectos y negocios en comunicación para impulsar la innovación y calidad de los contenidos y productos a fin de promover una cultura más humana.

2. Emprendimiento $y / 0$ vinculación profesional

FUENTE: UNIVERSIDAD ANÁHUAC MÉXICO, 2016. 
A lo largo del tiempo la actual Facultad de Comunicación ha mantenido su compromiso de calidad y pertinencia rediseñando su programa académico, el plan de estudios ha tenido un crecimiento sostenido en los 50 años de su existencia, y los egresados se colocan en posiciones estratégicas y de impacto social.

La actualización de los programas de estudio cada cinco años ha logrado que los egresados tengan los conocimientos necesarios para enfrentarse al campo laboral. Como características fundamentales del plan de estudio podemos resaltar:

- Está diseñado para que el alumno paulatinamente adquiera conocimientos en el ámbito de la administración, gestión y alta dirección de proyectos y empresas de entretenimiento.

- Cada materia, basada en su naturaleza y empleando las metodologías activas del modelo por competencias, orienta la evaluación del alumno para que aplique la teoría a la práctica.

- El diseño instruccional de los cursos de Practicum favorece el aprendizaje teórico en las aulas y el aprendizaje práctico en las empresas en donde el alumno realiza sus prácticas profesionales.

- El modelo de evaluación considera todas las particularidades de las asignaturas que fueron diseñadas exprofeso para la Licenciatura en Comunicación.

- El modelo educativo dentro del cual descasa el programa de estudios busca impulsar el autoestudio, trabajo colaborativo y multidisciplinario, uso de las TIC, formas de trabajo semipresenciales y a distancia, nuevas formas de emprendimiento social, así como dinámicas de trabajo con las que se encontrarán los alumnos al egresar.

- Como parte de una política institucional, la licenciatura ha incrementado sustantivamente el número y el monto de las becas asignadas a los alumnos en $35 \%$, así como el número de créditos educativos que a muchos estudiantes les permite asegurar su estadía en la institución.

- Diversas opciones de financiamiento y becas para los estudiantes.

- Reconocimiento del aprovechamiento escolar a través de los premios a la excelencia para cada semestre, en una ceremonia encabezada por el Rector de la Universidad. 


\section{CONCLUSIONES}

El vertiginoso cambio en la economía y en la tecnología en el mundo ha llevado a los países desde hace décadas a adaptarse a esta tendencia de globalización de servicios y saberes que incluyen la movilidad de personas entre países alrededor del mundo. De lo anterior surge la necesidad de adaptar los conocimientos impartidos en las aulas de clases a los requerimientos del mundo laboral tomando en cuenta el aspecto de la globalización.

La internacionalización del currículum permite que los estudiantes tengan contacto con una educación multicultural y desarrollen competencias que les permitirán tener una mayor formación competitiva.

En este sentido, la Facultad de Comunicación es una de las dos facultades en México acreditadas por el ACEJMC, lo que se traduce en un programa académico de calidad al nivel de universidades como la Universidad de Southern California y la Universidad de Texas en Austin, que están entre los cinco mejores programas de comunicación en el mundo de acuerdo con el Ranking Qs Communications \& Media (2019) y en mayores oportunidades de movilidad para los alumnos y los estudiantes.

La Universidad Anáhuac México consiente de los retos a los que se enfrentan los alumnos al egresar de las aulas actualiza los programas académicos tomando en consideración las mejores prácticas para la profesión y los comentarios de las acreditadoras, así como el bencbmarking con los programas de comunicación líderes en el mundo. De lo anterior se deriva que la Facultad esté a la vanguardia en México en cuanto a responder a las exigencias del contexto laboral nacional e internacional y mantiene su compromiso social al formar egresados líderes de acción positiva que basen sus acciones en el respeto a la dignidad de la persona, el desarrollo del ser humano y de la sociedad.

\section{REFERENCIAS}

Ackers, J. (1997). Evaluating UK courses: the perspective of the overseas student. En D. Mcnamara \& R. Harris (Eds.). Overseas Students in Higher Education: issues in teaching and learning. Routledge.

Bricall,J. (2000). Informe Universidad 2000. Conferencia de Rectores de las Universidades españolas (CRUE). https://www.observatoriuniversitari.org/es/files/20I4/05/Bricall_JM-200o-Informe-Universidad-2000.pdf

College Board. (2019). Trends in College Pricing 2019. https://research.collegeboard.org/pdf/ trends-college-pricing-2org-full-report.pdf 
Crisan-Mitra, C., y Borza, A. (2015). Internationalization In Higher Education. Conferencia Internacional Risk in Contemporary Economy, Galati, Rumania, Universidad de Galati. http://www. rce.feaa.ugal.ro/images/stories/RCE2015/Management-Marketing/MitraBorza.pdf

De Vita, G., y Case, P. (2003). Rethinking the internationalization agenda in UK higher education. Fournal of Further and Higher Education, 27(4), 383-398. https://doi.org/10.1080/ 0309877032000128082

Didou, S. (2005). Internacionalización y proveedores externos de Educación Superior en los países de América Latina y El Caribe: Principales Problemáticas. Departamento de Investigaciones Educativas; Centro de Investigación y de Estudios Avanzados. https://eco.mdp.edu.ar/cendocu/repositorio/oorig.pdf

Fallon, G., y Brown, R.B. (1999). What about the workers? Academic staff opinions about working with non-UK postgraduate students in higher education. Fournal of Further and Higher Education, 23, 4I-52. https://doi.org/I0.1080/0309877990230104

Gobierno de la República. (20I3). Plan Nacional de Desarrollo 20I3-20I8. https://www.snieg.mx/ contenidos/espanol/normatividad/MarcoJuridico/PND_20I3-20I8.pdf

IAU. (s.f.). Internationalization. https://www.iau-aiu.net/Internationalization?lang=en

INQAAHE. (2005). Biennial Conference Quality, Assurance \& DiversityConference, Wellington. https://www.inqaahe.org/events/inqaahe-conference-2005-wellington-new-zealand

Instituto de Educación Internacional. (202I). 2017 Project Atlas Infographics. https://www.iie.org/ en/Research-and-Insights/Project-Atlas/Explore-Data/Infographics/20I7-Project-Atlas-Infographics

Mazzarol, T., y Soutar, G. (1999). Sustainable competitive advantage for educational institutions: a suggested model. International Fournal of Educational Management, I3(6), 287- 300. https://doi. org/I0.IIO8/095I3549910294496

McNamara, D., y Harris, R. (Eds.). (1997). Overseas Students in Higher Education. Routledge.

OECD. (20I6). Education at a Glance: OECD indicators. OECD Publishing. https://www.oecd-ilibrary.org/education/education-at-a-glance-20I6_eag-20I6-en

OMC. (2000).Servicios de Enseñanza.https://www.wto.org/spanish/tratop_s/serv_s/education_s/ education_s.htm

QS Communication \& Media Ranking. (20I9). QS Top Universities. https://www.topuniversities. com/university-rankings/university-subject-rankings/2019/communication-media-studies

Rizvi, F. (2007). Internationalization of Curriculum: A Critical Perspective. SAGE Publications Ltd.

Secretaría de Gobernación. (2019). Plan Nacional de Desarrollo 2019-2024. http://www.dof.gob. $\mathrm{mx} /$ nota_detalle.php?codigo $=5565599 \& \mathrm{fecha}=\mathrm{I2} / 07 / 2019$

SEP. (2017). Sistema Educativo de los Estados Unidos Mexicanos: Principales Cifras 20I6-20I7. Secretaría de Educación Pública. https://www.planeacion.sep.gob.mx/Doc/estadistica_e_indicadores/principales_cifras/principales_cifras_2016_20I7_bolsillo.pdf 
SEVIS. (20I8). SEVIS by the numbers. https://studyinthestates.dhs.gov/20I8/05/check-out-the-latest-sevis-by-the-numbers-report

Tejedor, R., y Cervi, L. (20I7). Análisis de los estudios de Periodismo y Comunicación en las principales universidades del mundo. Competencias, objetivos y asignaturas. Revista Latina de Comunicación Social, 72(I), 626-I,647. https://doi.org/I0.4185/RLCS-20I7-I238

Tünnermann, C. (20I8). La internacionalización de la educación superior. Significado, relevancia y evolución histórica. En J. Gacel-Ávila (Coord.) Educación superior, internacionalización e integración en América Latina y el Caribe. Balance regional y prospectiva (p. 17-39). Unesco; IE SALC; UNC. http://erasmusplusriesal.org/sites/default/files/libro_la_es_inter_e_integracion_cres.pdf

Unesco. (1995). Documento de política para el cambio y el desarrollo en la educación superior. http://unesdoc.unesco.org/images/ooog/ooog89/og89g2s.pdf

Unesco. (1998). Educación Superior y Sociedad, 9(2). https://www.iesalc.unesco.org/ess/index.php/ ess3/issue/view/2I/2I

Unesco. (2009). Conferencia Mundial sobre la Educación Superior-2009: La nueva dinámica de la educación superior y la investigación para el cambio social y el desarrollo. http://www.unesco.org/education/WCHE2009/comunicado_es.pdf

Universidad Anáhuac México. (20I6). Plan de evaluación: perfil de egreso y resultados de aprendizaje. Universidad Anáhuac México.

Van Der Wende, M.C. (2000). Internationalising the curriculum: new perspectives and challenges. En B. Hudson y M.J. Todd (Eds.) Internationalising the Curriculum in Higher Education: reflecting on practice. Sheffield Hallam University Press.

Ziguras, C., y Rizvi, F. (200I). Future directions in international online education. En D. Davis \& D. Meares (Eds.) (pp.I5I-I64).Transnational Education: Australia Online. IDP Education Australia.

Esta obra está bajo Licencia Creative Commons Atribución-NoComercial-SinDerivadas 4.o Internacional. 